\title{
NATURAL SELECTION FOR QUANTITATIVE CHARACTERS
}

\author{
PETER O'DONALD* \\ Nuffield Unit of Medical Genetics, Department of Medicine, \\ University of Liverpool, P.O. Box 147, Liverpool L69 3BX, England
}

Received 10.iv.70

\section{INTRODUGTION}

DARWIN discovered natural selection because he realised that if individuals vary in certain characteristics then competition and the struggle for existence in the natural world must produce variations in their chances of survival. It is often said that this is a simple deduction from the facts of mortality, competition and variation. And yet the variations of a particular character may have no obvious effect on fitness. Is it obvious, for example, that Drosophila varying in the number of their sternopleural bristles will therefore vary in fitness? We could vary the intensity of competition in a population cage of Drosophila and yet have no idea how the fitness of individuals with different bristle numbers might vary with competition or indeed if it would vary at all. To infer that selection is operating on a character it is necessary to know that the character affects the outcome of competition or the chances of survival.

From the evidence of his notebooks, it seems clear that Darwin reached the idea of natural selection inductively. He observed, for example in the finches of the Galapagos Islands, that organisms of different species or subspecies are adapted to the particular ecological habitats they occupy. Thus the finches have beaks that are minutely adapted to their different feeding habits. Clearly one adaptation would permit the bird to feed more efficiently in one habitat than in another. If there are similar variations within a population of a single species, then some of the variations should be more efficient than others, and the individuals with the more efficient variations should have a higher fitness in the struggle for existence. The chain of reasoning is thus inductive and not deductive.

If we follow this chain of reasoning a little further, it seems that as long as any character affects the efficiency with which the organism lives in its habitat it should have some optimum form, or perhaps several different optimum forms. In general, if we can measure a character, a particular measurement or range of measurements should be the optimum-the value or values of the character at which it is most efficient. Normally, of course, if natural selection has done its job, we should expect that the optimum will be close to the mean of the population. A very clear example is shown by the variation of human weight at birth. New-born babies whose weight is near the mean weight of the population have a lower mortality than those who are much heavier or lighter than the mean. In this case the mortality does not increase much until the babies are considerably heavier or lighter than the mean. It will be shown later in this paper that there is a similar relationship between mortality and the bristle number of Drosophila.

To give a complete description of the natural selection of a quantitative character it is necessary to determine the mathematical relationship between

* Present address: Department of Genetics, Milton Road, Cambridge CB4 1XH. 
fitness and the character - the " fitness function " as I shall call it. Sometimes, as in the case of human birthweight, it is known empirically, for the mortality of babies in each range of weights can be obtained from hospital records. But often the only data are of the changes in the mean and variance before and after selection. It is then necessary to have some model of selection to fit the data to.

\section{Models of Natural SElection}

In natural selection, unlike the artificial selection of the plant and animal breeder, fitness probably takes a continuous range of values corresponding to the continuous range of values of the character. Discontinuities in the fitness function are unlikely. In artificial selection, however, the breeder may choose for breeding only those individuals whose values exceed some particular chosen value of the character. Thus all those who are less than the chosen value have a fitness of zero and the rest have a fitness of one. This is the case when the breeder is selecting for some improvement in an economically important character. In natural selection, however, the population may often be near its optimum and there may be little or no change in mean after selection. If, as in the case of human birthweight, the extreme individuals have a lower fitness than those round the mean, then the effect of selection is to reduce the variance and leave the mean unchanged. Of course, this can also be done by artificial selection by eliminating all individuals whose values lie outside two extreme values on opposite sides of the mean. As Van Valen $(1965,1967)$ has shown, it is possible to calculate what proportion of the tails of the distribution must be eliminated to produce any observed change of mean and variance.

A more realistic model is needed to obtain a general equation relating fitness to the character. The most obvious model, if selection is in favour of an optimum value of the character, is $w=1-\alpha-K(\theta-x)^{2}$ (O'Donald, 1968). In this model, the character $x$ has an optimum at $x=\theta$ and at this point the fitness is $w=1-\alpha$. Thus if fitness is determined solely by the chances of survival and not at all by fertility or fecundity, then $\alpha$ represents the proportion of random or non-selective deaths. The selective deaths are added on to the random deaths. An alternative model is when random and selective deaths are multiplicative so that $w=(1-\alpha)\left[1-K^{\prime}(\theta-x)^{2}\right]$. However, this model gives the same numerical results for it merely defines $K^{\prime}=K /(1-\alpha)$.

The model of $w=1-\alpha-K(\theta-x)^{2}$ has the advantage that fitness decreases only slowly while the character is close to the optimum but rapidly when it is a long way from it. There is a region round the optimum where fitness does not vary very much and a region well away from it where fitness is much lower and changes rapidly. This is the sort of relationship observed for human birthweight and Drosophila bristles. It has the further advantage that the changes of mean and variance can be predicted for a character $x$ with any frequency density $f(x)$. It is not necessary to assume, for example, that $x$ has the normal distribution. However, it has the disadvantage that negative fitnesses could theoretically occur, although they are impossible in practice. Suppose for example $x$ can take any value in the range $-\infty$ to $\infty$, as it could in a normal distribution. Then there will be some proportion of the population, perhaps an exceedingly small proportion in practice, that 
will have a negative fitness in the model. Of course, provided $\bar{w}$, the mean fitness, is several standard deviations away from the point of $w=0$, this proportion will be very small indeed and its effect quite negligible. However, since $w$ can never be negative, the distribution of $x$ can never be exactly normal, for it must always be truncated at the value of $x$ for which $w=0$. In practice, of course, the distribution can be very nearly normal in many cases.

O'Donald (1968) showed that if $w=1-\alpha-K(\theta-x)^{2}$, then after selection

$$
\begin{aligned}
\Delta \bar{x} & =\frac{2 V(\theta-\bar{x})-\mu_{3}}{\phi-(\theta-\bar{x})^{2}-V} \\
(\Delta \bar{x})^{2}+\Delta V & =\frac{V^{2}-\mu_{4}+2 \mu_{3}(\theta-\bar{x})}{\phi-(\theta-\bar{x})^{2}-V}
\end{aligned}
$$

where $\Delta \bar{x}=\bar{x}^{\prime}-\bar{x}$, the difference between the means before and after selection $\Delta V=V^{\prime}-V, \phi=(1-\alpha) / K$ and $V, \mu_{3}$ and $\mu_{4}$ are the second, third and fourth moments of $f(x)$ about the mean $\bar{x}$. If $x$ has the distribution

$$
g(x)=w f(x) / \bar{w}
$$

after selection, with mean and variance $\bar{x}^{\prime}$ and $V^{\prime}$, then $\Delta \bar{x}$ and $\Delta V$ are known. The moments of $f(x)$ can be calculated and hence there are two equations to estimate the two quantities $\phi$ and $\theta$ which determine the relative fitness of $x$.

\section{The intensity of selection}

The estimates of $\phi$ and $\theta$ can be used to calculate a quantity called the Intensity of Selection. It is defined (Van Valen, 1965) as

$$
I=\left(w_{0}-\bar{w}\right) / w_{0}
$$

where $w_{0}$ is the fitness of the optimum phenotype. Haldane (1954) originally defined the intensity of selection as

$$
I_{H}=\ln w_{0}-\ln \bar{w}
$$

so that $I$ is related to $I_{H}$ by the equation

$$
I=1-e^{-I_{H}} \text {. }
$$

For small values of $I_{H}, I=I_{H}$ approximately. If the distribution of $x$ is normal before and after selection, a simple expression can then be derived for $I_{H}$ in terms of the changes of mean and variance. But Haldane's definition has no other advantages. The definition of $I=\left(w_{0}-\bar{w}\right) / w_{0}$ is the same as for the genetic load, except that here we are dealing with phenotypes, and not genotypes alone. The intensity of selection could equally well be called the "phenotypic load ": it is the proportion of the population eliminated by selection because not all individuals have the optimum value of the character.

The model $w=1-\alpha-K(\theta-x)^{2}$ may be called simply the "quadratic model "since fitness decreases as the square of the deviation from the optimum. In this model

$$
\begin{aligned}
& w_{0}=1-\alpha \\
& \bar{w}=1-\alpha-K(\theta-\bar{x})^{2}-K V .
\end{aligned}
$$


Thus

$$
\begin{aligned}
I & =\frac{K(\theta-\bar{x})^{2}+K V}{1-\alpha} \\
& =\frac{(\theta-\bar{x})^{2}+V}{\phi}
\end{aligned}
$$

We have in general (O’Donald, 1968) the values

$$
\begin{aligned}
\theta-\bar{x} & =\frac{V^{2}(\Delta \bar{x})-\mu_{4}(\Delta \bar{x})+\mu_{3}\left[(\Delta \bar{x})^{2}+\Delta V\right]}{2 V\left[(\bar{x})^{2}+\Delta V\right]-2 \mu_{3}(\Delta \bar{x})} \\
\phi & =\frac{2 V(\theta-\bar{x})-\mu_{3}}{\Delta \bar{x}}+(\theta-\bar{x})^{2}+V
\end{aligned}
$$

and hence $I$.

Often, however, $\Delta \bar{x}$ will not differ significantly from zero if the population has been subjected to selection for a long time. If $\Delta \bar{x}=0$, then $\theta-\bar{x}=\mu_{3} / 2 V$ and using the change in $V$ to estimate $\phi$ we have

$$
\phi=\frac{V^{2}-\mu_{4}+\mu_{3}^{2} / V}{\Delta V}+\frac{\mu_{3}}{2 V}+V
$$

In this case if $f(x)$ is a normal distribution we have $\mu_{3}=0, \mu_{4}=3 V^{2}$ and

$$
I=\frac{V-V^{\prime}}{3 V-V^{\prime}}
$$

Given values of $\phi$ and $\theta$, an equation for the relative fitness of $x$ can be calculated. Since we have defined $\phi=(1-\alpha) / K, \phi=1 / K$ when $\alpha=0$. Thus $w=1-(\theta-x)^{2} / \phi$. The individuals at the optimum then have a relative fitness of one.

If the actual proportion of deaths, $D$, is known for the period of selection, then the absolute fitnesses can be calculated; for we have

or

$$
D=1-\bar{w}
$$$$
\bar{w}=1-D \text {. }
$$

Thus

$$
1-D=1-\alpha-K(\theta-\bar{x})-K V \text {. }
$$

But

$$
I=\frac{(\theta-\bar{x})^{2}+V}{\phi}
$$

so that

$$
\begin{aligned}
1-D & =1-\alpha-K I \phi \\
& =(1-\alpha)(1-I) .
\end{aligned}
$$

Therefore

$$
\alpha=\frac{D-I}{1-I}
$$

and hence $K=(1-\alpha) / \phi$ from the definition of $\phi$. Thus $\alpha, K$ and $\theta$ can all be calculated and hence the fitness function

$$
w=1-\alpha-K(\theta-x)^{2}
$$

obtained. 


\section{Other models of the fitness function}

The quadratic model is but one possible model of the fitness function. Obviously there are infinitely many of such models. A general model might take the form

$$
w=1-\alpha-\beta x-\gamma x^{2}-\delta x^{3}-\ldots
$$

but it would involve too many constants to be fitted to actual data. A possible model involving only two constants is

$$
w=1-\alpha-K|\theta-x| \text {. }
$$

Here fitness decreases linearly on either side of the optimum. A fitness function having a sharp discontinuous peak at the optimum may be thought to be unlikely to be found in practice. It has other disadvantages. The mean fitness is by definition

$$
\bar{w}=\int_{-\infty}^{\infty}[1-\alpha-K / \theta-x /] f(x) d x .
$$

The distribution after selection is

$$
\begin{aligned}
g(x) & =w f(x) / \bar{w} \\
& =[1-\alpha-K / \theta-x /] f(x) / \bar{w}
\end{aligned}
$$

and therefore the mean and variance after selection are defined by

$$
\begin{aligned}
& \bar{x}^{\prime}=\frac{\int_{-\infty}^{\infty} x[1-\alpha-K / \theta-x /] f(x) d x}{\bar{w}} \\
& V^{\prime}=\frac{\int_{-\infty}^{\infty} x^{2}[1-\alpha-K / \theta-x /] f(x) d x}{\bar{w}}-\left(\bar{x}^{\prime}\right)^{2} .
\end{aligned}
$$

These integrals can be evaluated if $f(x)$ is a normal distribution. Solutions have been obtained in terms of incomplete gamma functions but calculations of the intensity of selection are then difficult.

\section{Diregt galqulations of fitness}

Dowdeswell (1961) obtained data on the distributions of the spots on the underside of the hind wings of the butterfly Maniola jurtina. He reared larvae and obtained the distribution of spots. In this sample there had been no adult mortality. He also obtained the distribution of spots of individuals flying in the wild. These may have been subject to natural selection after their emergence. Thus the distribution of the sample of reared butterflies is the distribution before selection and the distribution of butterflies flying wild is the distribution after selection. The distributions of males before and after selection were similar, but the distributions of the females differed and selection had presumably been at work among them. 
The data on the distributions of spotting of the females are shown in table 1. With data such as these, the relative fitnesses can be calculated directly without the need to use any theoretical model of selection. But first consider

TABLE 1

$\begin{array}{ccc}\begin{array}{c}\text { Numbers } \\ \text { of spots }\end{array} & \text { Before } & \begin{array}{c}\text { After } \\ \text { selection } \\ \text { selection }\end{array} \\ 0 & f & f^{1} \\ 1 & 124 & 294 \\ 2 & 67 & 111 \\ 3 & 34 & 53 \\ 4 & 10 & 13 \\ \text { Totals } & 2 & 0 \\ & 237 & 471\end{array}$

the case when the individuals after selection are the survivors of the individuals before selection, that is when the populations before and after selection are known. The absolute and relative fitnesses can then be calculated for each phenotypic value, as shown in table 2 .

TABLE 2

$\begin{array}{ccccc}\begin{array}{c}\text { Phenotypic } \\ \text { values }\end{array} & \begin{array}{c}\text { Frequency } \\ \text { before selection }\end{array} & \begin{array}{c}\text { Frequency } \\ \text { after selection }\end{array} & \begin{array}{c}\text { Absolute } \\ \text { fitness }\end{array} & \begin{array}{c}\text { Relative } \\ \text { fitnéss }\end{array} \\ x & f & f^{1} & w_{\text {abs }} & w_{\text {rel }} \\ 0 & u_{0} & u_{0}^{1} & u_{0}^{1} / u_{0} & 1 \\ 1 & u_{1} & u_{1}^{1} & u_{1}^{1} / u_{1} & u_{1}^{1} u_{0} / u_{1} u_{0}^{1} \\ 2 & u_{2} & u_{2}^{1} & u_{2}^{1} / u_{2} & u_{2}^{1} u_{0} / u_{2} u_{0}^{1} \\ 3 & u_{3} & u_{3}^{1} & u_{3}^{1} / u_{3} & u_{3}^{1} u_{0} / u_{3} u_{0}^{1} \\ 4 & u_{4} & u_{4}^{1} & u_{4}^{1} / u_{4} & u_{4}^{1} u_{0} / u_{4} u_{0}^{1}\end{array}$

If, however, the numbers $f$ and $f^{1}$ are of samples taken from the populations before and after selection, as in the case of Dowdeswell's data, then only relative fitnesses can be calculated, for the actual frequencies before and after selection are determined by the sizes of the samples and not by the

TABLE 3

$\begin{array}{cccc}x & f & w_{\text {rel }} & \mathrm{P}=f / 237 \\ 0 & 124 & 1.0000 & 0.5232 \\ 1 & 67 & 0.6987 & 0.2827 \\ 2 & 34 & 0.6575 & 0.1435 \\ 3 \text { and } 4 & 12 & 0.4569 & 0.05063\end{array}$

chances of survival. This method of calculating relative fitness is an extension of Woolf's (1955) for estimating the relative fitness of two phenotypes. The fitnesses of individuals with different spot numbers calculated from Dowdeswell's data are given in table 3. The mean fitness is therefore

$$
\begin{aligned}
\bar{w} & =\sum w P \\
& =0.8382 .
\end{aligned}
$$


Since we have defined the maximum fitness to be $w=1$, the intensity of selection is

$$
\begin{aligned}
I & =1-\bar{w} \\
& =0.1618 .
\end{aligned}
$$

This value of $I$ is likely to be too large since the large standard errors in the estimates of $P$ and $P^{1}$, the proportions of individuals of a given spot number before and after selection, will inflate the value of $I$. But the intensity of selection may also be calculated by the quadratic model. Using the formulae given by Fisher (1954) we calculate

$$
\begin{aligned}
& s_{1}=\sum x f=173 \\
& s_{2}=\sum x^{2} f=325 \\
& s_{3}=\sum x^{3} f=737 \\
& s_{4}=\sum x^{4} f=1933
\end{aligned}
$$

and then

$$
\begin{aligned}
& S_{2}=s_{2}-\frac{1}{n} s_{1}^{2}=198 \cdot 7173 \\
& S_{3}=s_{3}-\frac{3}{n} s_{2} s_{1}+\frac{2}{n^{2}} s_{1}^{3}=209 \cdot 6532 \\
& S_{4}=s_{4}-\frac{4}{n} s_{3} s_{1}+\frac{6}{n^{2}} s_{2} s_{1}^{2}-\frac{3}{n^{3}} s_{1}^{4}=618.2545
\end{aligned}
$$

to obtain the $k$ statistics

$$
\begin{aligned}
& k_{1}=\frac{1}{n} s_{1}=0.72996 \\
& k_{2}=\frac{1}{(n-1)} S_{2}=0.84202 \\
& k_{3}=\frac{n}{(n-1)(n-2)} S_{3}=0.89592 \\
& k_{4}=\frac{n(n+1)}{(n-1)(n-2)(n-3)} S_{4}-\frac{3}{(n-2)(n-3)} S_{2}^{2}=0.53287 .
\end{aligned}
$$

The $k$ statistics give the unbiassed estimates of the moments

$$
\begin{aligned}
\bar{x} & =k_{1}=0.72996 \\
V & =k_{2}=0.84202 \\
\mu_{3} & =k_{3}=0.89592 \\
\mu_{4} & =k_{4}+3 k_{2}^{2}=2 \cdot 65987 .
\end{aligned}
$$


After selection we find

so that

$$
\begin{aligned}
\bar{x}^{1} & =0.54352 \\
V^{1} & =0.57629
\end{aligned}
$$

$$
\begin{aligned}
& \Delta \bar{x}=-0 \cdot 18643 \\
& \Delta V=-0.26573 .
\end{aligned}
$$

There is no question of the significance of these changes of mean and variance. For the difference $\Delta \tilde{x}, t=2 \cdot 870$ and $F=V / V^{1}=1 \cdot 461$. These values of $t$ and $F$ are very significant. Thus we estimate

$$
\begin{aligned}
\theta-\bar{x} & =\frac{(\Delta \bar{x})\left(V^{2}-\mu_{4}\right)+\mu_{3}\left[(\Delta \bar{x})^{2}+\Delta V\right]}{2 V\left[(\Delta \bar{x})^{2}+\Delta V\right]-2 \mu_{3}(\Delta \bar{x})} \\
& =-2.8555 ;
\end{aligned}
$$

which is a curious, though not necessarily unreasonable, value; since it makes $\theta=-2 \cdot 1255$. However, we then get

and

$$
\begin{aligned}
\phi & =\frac{2 V(\theta-\bar{x})-\mu_{3}}{\Delta \bar{x}}+(\theta-\bar{x})^{2}+V \\
& =39.5943,
\end{aligned}
$$

This is, of course, too high because the observed value of $I$, calculated directly was only $0 \cdot 1618$. But this observed value was calculated assuming that $x=0$ was the optimum phenotype. If negative values of $x$ were possible and the optimum were then at $-2 \cdot 1$, the value of $I$ would necessarily be greater. The standard errors of the estimates of $I$ are also large; and, indeed, a rough calculation of the standard errors suggests that the values of $I$ do not differ significantly.

Given the estimates of $\theta$ and $\phi$ it is possible to obtain the relative fitness function. Letting $\alpha=0$ so that $w=1$ when $x=\theta$, we get $K=1 / \phi=$ $0 \cdot 025256$. Thus

$$
\begin{aligned}
w & =1-K(\theta-x)^{2} \\
& =1-0.025256(-2 \cdot 1255-x)^{2} \\
& =0.88590-0.10736 x-0.025256 x^{2} .
\end{aligned}
$$

The relative fitnesses calculated directly and by the quadratic model are compared in table 4 . The relative fitness in the quadratic model is always

TABLE 4

$\begin{array}{ccc}x & w_{\text {rel }}(\text { direct) } & w_{\text {rel }} \text { (quadratic model) } \\ 0 & 1.0000 & 0.8859 \\ 1 & 0.6987 & 0.7533 \\ 2 & 0.6575 & 0.5702 \\ 3 \text { and } 4 & 0.4569 & 0.2892\end{array}$

less than 1 , since the optimum fitness would occur at the point $x=-2 \cdot 1255$, an impossible phenotype on the scale of measurement of the character. But 
it is of course quite possible that selection is acting on some underlying physiological character, of which spot number is but a partial manifestation, and the optimum of the physiological character might well correspond to a spot number of $-2 \cdot 1255$, which, however, cannot be phenotypically observed in terms of spots. If we give the phenotype $x=0$ a relative fitness of $w_{\text {rel }}=$ 1.0000 in both models, we get the values shown in table 5.

TABLE 5

$\begin{array}{ccc}x & w_{\text {rel }} \text { (direct) } & w_{\text {rel }} \text { (quadratic model) } \\ 0 & 1.0000 & 1.0000 \\ 1 & 0 \cdot 6987 & 0.8503 \\ 2 & 0.6575 & 0.6436 \\ 3 \text { and } 4 & 0.4569 & 0.3265\end{array}$

Note that by the quadratic model

$$
\begin{aligned}
w_{\text {rel }} & =1.0000-\frac{0.10736}{0.88590} x-\frac{0.025256}{0.88590} x^{2} \\
& =1.0000-0.12119 x-0.028508 x^{2} .
\end{aligned}
$$

The corresponding intensities of selection are then

$$
\begin{aligned}
I(\text { direct }) & =0 \cdot 1618 \\
I(\text { quadratic model }) & =0 \cdot 1275 .
\end{aligned}
$$

This last figure is a very reasonable one. We should expect it to be less than the direct estimate since it will not be inflated by chance variations in the proportions of the phenotypes observed before and after selection.

\section{Measuring the selection for bristle number in Drosophilia}

An experiment was carried out to determine the selection operating on adult Drosophila melanogaster differing in the number of their sternopleural bristles. It was designed to test Darwin's idea that the intensity of competition determines the intensity of the selection.

Adult Drosophila were put in population cages in which the amount of food was varied. As many as 25 food tubes could be screwed on to each population cage. One cage had all 25 tubes. Two others had only eight tubes. About 1800 Drosophila in approximately equal numbers of males and females were put in the cages. In the cages with only eight food tubes, the tubes were thick with Drosophila apparently fighting for the food. In the cage with all 25 tubes, life was more orderly and only a few Drosophila at a time entered a food tube to feed. Throughout the experiments eggs were continually being laid and the food tubes were changed once the larvae had started to grow to an appreciable size.

The cages were left until about half or two-thirds of the flies were dead. They had been counted for their bristle number before they were put in the cages and they were then counted again. In the cage with all 25 food tubes, the flies lived for about 3 weeks before they began to die off in considerable numbers. The survivors were counted 4 weeks after the count of the original population. In the cages with only eight food tubes, death was rapid and about 65 per cent. had died after only 6 days. 
There was no significant selection of the flies in the cage with 25 food tubes (cage Gl). Only the males showed a possible selective effect. The fitnesses of individuals near the mean were somewhat higher than the fitnesses of individuals well away from it. But this effect was not statistically significant.

The results were different in cage $G 2$ when the food was reduced to only eight tubes. There was no significant selection of the females $\left(\chi^{2}=\right.$ 8.6270 for 9 degrees of freedom) but highly significant selection of the males. The data are given in table 6. Adding together the frequencies of bristle

TABLE 6

\begin{tabular}{|c|c|c|c|}
\hline \multicolumn{4}{|c|}{ Frequencies of males } \\
\hline $\begin{array}{c}\text { Bristle } \\
\text { number } \\
x\end{array}$ & $\begin{array}{c}\text { Before } \\
\text { selection } \\
f\end{array}$ & $\begin{array}{c}\text { After } \\
\text { selection } \\
f^{\beth}\end{array}$ & $\begin{array}{c}\text { Absolute } \\
\text { fitness } \\
f^{1} / f\end{array}$ \\
\hline 12 & 1 & 0 & \\
\hline 13 & 1 & 0 & \\
\hline 14 & 25 & 7 & $0 \cdot 1923$ \\
\hline 15 & 51 & 8 & \\
\hline 16 & 98 & 45 & 0.4592 \\
\hline 17 & 104 & 36 & $0 \cdot 3462$ \\
\hline 18 & 106 & 32 & $0 \cdot 3019$ \\
\hline 19 & 67 & 22 & $0 \cdot 3284$ \\
\hline 20 & 54 & 17 & \\
\hline 21 & 24 & 6 & \\
\hline 22 & 21 & 4 & \\
\hline 23 & 7 & 1 & 0.2613 \\
\hline 24 & 4 & 1 & \\
\hline 25 & 0 & 0 & \\
\hline 26 & 1 & 0 & \\
\hline Totals & 564 & 179 & $0 \cdot 3174$ \\
\hline
\end{tabular}

numbers up to 14 and above 21 , we get $\chi^{2}=19 \cdot 438$ for 8 degrees of freedom, which corresponds to a probability of 1.34 per cent. And it is obvious from the data that individuals with fewer than 16 bristles or more than 19 bristles have much lower fitnesses than those with 16 to 19 bristles. As the model requires, selection is acting in favour of an optimum in the range 16-19.

Calculating the moments as before, we find (taking 18 as origin to simplify the calculation)

and

$$
\begin{aligned}
s_{1} & =-166 \\
s_{2} & =2634 \\
s_{3} & =536 \\
s_{4} & =36030 \\
S_{2} & =2585 \cdot 142 \\
S_{3} & =2833 \cdot 005 \\
S_{4} & =38017 \cdot 406
\end{aligned}
$$


so that

$$
\begin{aligned}
& k_{1}=0.294326 \\
& k_{2}=4.591726 \\
& k_{3}=5.049888 \\
& k_{4}=4.659676 .
\end{aligned}
$$

The moments are therefore

$$
\begin{aligned}
& \bar{x}=17 \cdot 70567 \\
& V=4.59173 \\
& \mu_{3}=5.04989 \\
& \mu_{4}=67.91152 \text {. } \\
& \text { After selection } \quad \bar{x}^{1}=17.56983 \\
& \mathrm{~V}^{\mathbf{1}}=3 \cdot 57234 \\
& \Delta V=-1 \cdot 01938 \text {. }
\end{aligned}
$$$$
\text { so that } \Delta \bar{x}=-0 \cdot 13584
$$

The ratio $V / V^{1}$ is highly significant and shows that selection has reduced the variance. However $\Delta \bar{x}$ does not differ significantly from zero $(t=0 \cdot 8718)$.

Since we cannot use the value of $\Delta \bar{x}$ to fit the quadratic model, we must put $\Delta \bar{x}=0$ and get

$$
\begin{aligned}
\theta-\bar{x} & =\mu_{3} / 2 V \\
& =0.54989 .
\end{aligned}
$$

Then the change in variance can be used to estimate $\phi$. This gives

$$
\begin{aligned}
\phi & =\frac{V^{2}-\mu_{4}+2 \mu_{3}(\theta-\bar{x})}{\Delta V}+(\theta-\bar{x})^{2}+V \\
& =45.383 .
\end{aligned}
$$

From these values, $I=0 \cdot 1078$.

The equation for the fitness function can now be obtained, for

$$
\text { giving } \quad \begin{aligned}
\bar{w} & =179 / 564=0.317375 \\
D & =0.682625 \\
\text { and } \quad & \begin{aligned}
\alpha & =\frac{D-I}{1-I} \\
& =0.64426 .
\end{aligned}
\end{aligned}
$$

Then $K=(1-\alpha) / \phi=0.0078385$.

The fitness function is therefore

$$
\begin{aligned}
w & =1-\alpha-K(\theta-x)^{2} \\
& =-2.2566+0.28619 x-0.0078385 x^{2}
\end{aligned}
$$


The observed and predicted fitness can now be compared. They are given in table 7. To check the numerical accuracy of the fit we must have

$$
\begin{aligned}
\bar{w}_{\text {pred icted }}=\bar{w}_{\text {observed }} \text { and } \bar{w}_{\text {predicted }} & =\sum f w_{\text {predicted }} / 564 \\
& =179.033 / 564 \\
& =0.31743
\end{aligned}
$$

\begin{tabular}{|c|c|c|c|c|}
\hline$x$ & $f$ & $w_{\text {predicted }}$ & & $w_{\text {observed }}$ \\
\hline 12 & 1 & 0.0490 & & \\
\hline 13 & 1 & $0 \cdot 1392$ & & \\
\hline 14 & 25 & 0.2138 & $0 \cdot 2492$ & 0.1923 \\
\hline 15 & 51 & 0.2727 & & \\
\hline 16 & 98 & 0.3159 & & 0.4592 \\
\hline 17 & 104 & 0.3434 & & $0 \cdot 3462$ \\
\hline 18 & 106 & $0 \cdot 3552$ & & 0.3019 \\
\hline 19 & 67 & 0.3514 & & 0.3284 \\
\hline 20 & 54 & 0.3319 & & \\
\hline 21 & 24 & 0.2967 & & \\
\hline 22 & 21 & $0 \cdot 2458$ & & \\
\hline 23 & 7 & $0 \cdot 1793$ & 0.2859 & 0.2613 \\
\hline 24 & 4 & 0.0971 & & \\
\hline 25 & 0 & -0.0008 & & \\
\hline 26 & 1 & -0.1140 & & \\
\hline
\end{tabular}

which is a sufficiently close numerical agreement. Since the model does give phenotypes with negative fitnesses, which are impossible, a small error will be introduced. But it only affects one individual out of 564 and will therefore be negligible.

The other cage (G3) with only eight food tubes gave different results. In the first place there was significant selection of both males and females. The data of the males are given in table 8 and those of the females in table 9 .

\begin{tabular}{rrrl}
\multicolumn{5}{c}{ Table 8} \\
\multicolumn{4}{c}{ Males } \\
$x$ & $f$ & $f^{1}$ & $f^{1} / f$ \\
$\leqslant 14$ & 49 & 19 & $0 \cdot 388$ \\
15 & 76 & 25 & $0 \cdot 329$ \\
16 & 122 & 63 & $0 \cdot 516$ \\
17 & 102 & 33 & $0 \cdot 324$ \\
18 & 102 & 51 & $0 \cdot 500$ \\
19 & 67 & 35 & $0 \cdot 522$ \\
$\geqslant 20$ & 87 & 27 & $0 \cdot 310$ \\
Totals & 605 & 253 & $0 \cdot 418$
\end{tabular}

Selection is highly significant on the males $\left(\chi^{2}=21 \cdot 221\right.$ for 6 degrees of freedom) and significant at the 2 per cent. level on females $\left(\chi^{2}=15.266\right.$ for 6 degrees of freedom).

In both males and females the fitness function appears to be bi-modal. Males with 17 bristles have a low relative fitness compared to those with 16 and 18 and 19 bristles. Females with 18 bristles have a low relative fitness compared to those with 16 and 17 and 19 bristles. This is a most unexpected result and different from the results of the other cages. There can 


\begin{tabular}{|c|c|c|c|}
\hline \multicolumn{4}{|c|}{$\mathrm{T}_{\mathrm{ABLE}} 9$} \\
\hline$x$ & $f$ & $f^{1}$ & $f^{1} / f$ \\
\hline$\leqslant 15$ & $\begin{array}{l}65 \\
88\end{array}$ & $\begin{array}{l}35 \\
49\end{array}$ & $\begin{array}{l}0.538 \\
0.557\end{array}$ \\
\hline 17 & 116 & 70 & 0.603 \\
\hline 18 & 122 & 50 & 0.410 \\
\hline 19 & 69 & 39 & 0.565 \\
\hline 20 & 54 & 21 & 0.389 \\
\hline$\geqslant 21$ & 62 & 27 & 0.435 \\
\hline Totals & 576 & 291 & 0.505 \\
\hline
\end{tabular}

be no doubt that selection was acting differently in cages G2 and G3. The mean fitnesses were significantly higher in G3 although the flies were kept for the same period- 6 days-in both cages. Comparing the males in the two cages we have

$\begin{array}{lcccc} & f^{1} & f_{-} f^{\mathbf{1}} & f & \bar{w}=f^{\mathbf{1}} / f \\ \text { Cage G2 } & 179 & 385 & 564 & 0 \cdot 3174 \\ \text { Cage G3 } & 253 & 352 & 605 & 0 \cdot 4182 \\ \text { Totals } & 432 & 737 & 1169 & -\end{array}$

and $\chi^{2}=12 \cdot 302$ for 1 degree of freedom, which is very significant with $\mathrm{P}<0 \cdot 001$. Comparing females

$\begin{array}{lcccc} & f^{\mathbf{1}} & f_{-} f^{\mathbf{1}} & f & \bar{w} \\ \text { Cage G2 } & 277 & 364 & 641 & 0 \cdot 4321 \\ \text { Cage G3 } & 291 & 285 & 576 & 0.5052 \\ \text { Totals } & 568 & 649 & 1217 & -\end{array}$

and $x^{2}=6.218$ for 1 degree of freedom, which is also significant with $0.02>\mathrm{P}>0.01$. For some reason, therefore, the mortality was different in the two cages although the populations were subject to similar competition for food for the same periods of time.

\section{EFFEGT ON fitness of A NEW MUtATion}

Suppose a mutation has a given average effect on bristle numbers. Suppose for example that it increases the number of bristles by two on average. Assuming its effects are additive, what will its selective coefficient be? In general let its average effect be $d$. Then from the fitness function

$$
w_{1}=-2 \cdot 2566+0 \cdot 28619(x+d)-0.0078385(x+d)^{2} .
$$

Hence the mean fitness of such a gene, assuming it appears among the individuals of the population at random, is

$$
\bar{w}_{1}=\frac{1}{n} \sum w_{1} f .
$$

In general we showed that

$$
\bar{w}=1-\alpha-K(\theta-\bar{x})^{2}-K V
$$


and therefore in the case of a gene with an average effect $d$, its mean fitness will be

$$
\bar{w}_{1}=-2 \cdot 2566+0 \cdot 28619(\bar{x}+d)-0.0078385(\bar{x}+d)^{2}-K V
$$

where $K V=0.0078385 \times 4.5917$ and hence

$$
\bar{w}_{1}=2 \cdot 2926+0.28619(\bar{x}+d)-0.0078385(\bar{x}+d) .
$$

Table 10 shows the relative fitnesses and selective coefficients of mutations

\begin{tabular}{|c|c|c|c|}
\hline $\begin{array}{c}\text { Average effect } \\
\text { of mutation }\end{array}$ & Fitness & $\begin{array}{c}\text { Relative fitness } \\
\text { of mutation }\end{array}$ & $\begin{array}{l}\text { Selective } \\
\text { coefficient }\end{array}$ \\
\hline$d$ & $\bar{w}_{1}$ & & \\
\hline 0 & 0.31737 & $1 \cdot 00000$ & 0 \\
\hline $0 \cdot 25$ & $0 \cdot 31904$ & $1 \cdot 00525$ & $-0 \cdot 00525$ \\
\hline 0.5 & 0.31972 & $1 \cdot 00741$ & -0.00741 \\
\hline 0.75 & 0.31943 & $1 \cdot 00648$ & -0.00648 \\
\hline 1.00 & $0 \cdot 31815$ & $1 \cdot 00246$ & -0.00246 \\
\hline $2 \cdot 00$ & $0 \cdot 30326$ & 0.95533 & $0 \cdot 0444$ \\
\hline 5.00 & $0 \cdot 16451$ & 0.51835 & 0.482 \\
\hline
\end{tabular}
having different average effects.

The larger the effect of the mutation, the lower its mean fitness: only for values of $d$ between 0 and 1 does it possess a selective advantage, and its selective advantage in this range is quite small. The reason for this is, of course, that the population is stabilised near its optimum. It clearly illustrates Fisher's point (1930) that only mutations having small effects can be advantageous.

The converse is also interesting. Mutations having large effects will be disadvantageous-indeed they will be extremely disadvantageous, and thus rapidly eliminated. The major contribution to the variance must therefore come from mutations with small effects and they will have very small selective coefficients. If, therefore, the many polymorphisms that have been discovered among isozymes affect quantitative characters, they will generally be subject to only weak selection. However, they will not be truly neutral, as has often been suggested, although the selection may be much too weak to be detected.

A similar calculation can be carried out for mutations affecting the spotting of Maniola jurtina. It was shown that if individuals with no spots are given a relative fitness of one then for any spot number $x$ the fitness is given by

$$
w=1 \cdot 0000-0.12119 x-0.028508 x^{2}
$$

The mean fitness of a mutation with an average effect $d$ is therefore

$$
\begin{aligned}
\bar{w}_{1} & =1 \cdot 0000-0.12119(\bar{x}+d)-0.028508(\bar{x}+d)^{2}-0.028508 \mathrm{~V} \\
& =0.97600-0.12119(\bar{x}+d)-0.028508(\bar{x}+d)^{2} .
\end{aligned}
$$

Table 11 shows the fitnesses and selective coefficients of mutations having different average effects on spotting. As with the bristles, so it is with the spots: the selective coefficients are fairly small if the effect of the mutation is small. Only if the mutation has a very large effect will its selective coeffi- 
cient also be large. This shows that the selective coefficients of individual genes cannot be inferred from the relative fitnesses of the phenotypes. There may be very large variations in fitness among the phenotypes, but if the character is determined by many genes with small effects each particular gene will have only a small selective advantage or disadvantage. The extreme phenotypes will always be very disadvantageous because they are largely homozygous either for genes that increase the value of the character or for genes that decrease it; such phenotypes are, of course, rare in the population.

TABLE 11

$\begin{array}{cccl}\begin{array}{c}\text { Average effect } \\ \text { of mutation }\end{array} & \begin{array}{c}\text { Fitness } \\ d\end{array} & \begin{array}{c}\text { Relative fitness } \\ \text { of mutation }\end{array} & \begin{array}{c}\text { Selective } \\ \text { coefficient }\end{array} \\ 0 & \bar{w}_{1} & & \\ 0 \cdot 25 & 0 \cdot 8723 & 1 \cdot 0000 & 0 \\ 0 \cdot 5 & 0 \cdot 8332 & 0 \cdot 9551 & 0 \cdot 0449 \\ 0 \cdot 75 & 0 \cdot 7826 & 0 \cdot 8971 & 0 \cdot 1029 \\ 1 \cdot 00 & 0 \cdot 7342 & 0 \cdot 8416 & 0 \cdot 1584 \\ 2 \cdot 00 & 0 \cdot 6793 & 0 \cdot 7787 & 0 \cdot 2213 \\ & 0.4327 & 0 \cdot 4960 & 0 \cdot 5040\end{array}$

The number of loci involved in the determination of a quantitative character can be calculated if some unlikely assumptions are made. Consider the character of spotting on Maniola jurtina. Assume that it is determined by a number of loci at each of which two alleles segregate. Assume that the gene frequencies are the same at all the loci and that each locus has the same average effect. We then have at each locus the following genotypes, effects and genotypic frequencies:

$\begin{array}{ccc} & \text { Effect on } & \\ \text { Genotypes } & \text { spot number } & \text { Frequency } \\ A A & d & p^{2} \\ A a & 0 & 2 p q \\ a a & -d & q^{2}\end{array}$

The cumulants of the distribution are then

$$
\begin{aligned}
& K_{1}=n(p-q) d \\
& K_{2}=2 n p q d^{2} \\
& K_{3}=-2 n p q(p-q) d^{3} \\
& K_{4}=2 n p q(1-6 p q) d^{4} .
\end{aligned}
$$

The first cumulant cannot be estimated from the sample, for it has been measured arbitrarily from the mid-point of the homozygotes. However, the second, third and fourth cumulants do not depend on the point of origin. It can be shown (O'Donald, to be published) that when the gene frequencies vary at different loci the estimate obtained of the number of loci will be too low if the mean gene frequency is close to zero or one but too high if it is about $0 \cdot 5$. For mean gene frequencies of $0 \cdot 5,0$ and 1 , the error is about 30 per cent. If the mean gene frequency is about $0 \cdot 2$, the error in the estimate of the number of loci is negligible. 
Equating the cumulants to the values obtained for the $k$ statistics we have

$$
\begin{aligned}
2 n p q d^{2} & =0.84202 \\
-2 n p q(p-q) d^{3} & =0.89592 \\
2 n p q(1-6 p q) d^{4} & =0.53287 . \\
K_{3}^{2} & =4 n^{2} p^{2} q^{2}(1-4 p q) d^{6} .
\end{aligned}
$$

But

Therefore

$$
\frac{K_{3}^{2}}{K_{4} K_{2}}=\frac{1-4 p q}{1-6 p q}=1 \cdot 7889,
$$

which gives the value $2 p q=0.23433$. Also $K_{4} / K_{2}=(1-6 p q) d^{2}=0.63285$, giving $d=1.4597$ and $n=1.7$. Since $n$ must be an integer we can put $n=2$ for which $d=1 \cdot 3404$. A gene with this average effect would have a selective coefficient of $0 \cdot 309$. It would rapidly be eliminated from a population or maintained at a very low frequency by mutation.

A similar calculation can be done for the bristle numbers of Drosophila. We have

$$
\frac{1-4 p q}{1-6 p q}=1 \cdot 19188
$$

and $2 p q=0 \cdot 26178, d=1 \cdot 1245$ and $n=24$. Since some extremely simple assumptions were made to calculate these values, they can be regarded only as suggesting the orders of magnitude of the true values. They may then be used to give some idea of how rapidly a character might change as an advantageous mutation spreads through a population. If, for example, there are only two loci to determine the number of spots on the wings of Maniola jurtina, then a mutation reducing spotting by one spot could spread very rapidly. There is indeed some experimental evidence of great instability in the spot distributions of Maniola jurtina in south-west England (Creed, Dowdeswell, Ford and McWhirter, 1959).

But as the calculations also show, a wide range of variation in the fitness of the phenotypes will not necessarily produce rapid evolution. It certainly will not do so if the population is near its optimum and the phenotypes are determined by many genes each with small effects. The mutations affecting the bristle numbers of Drosophila, for example, will be advantageous only if they have small effects and very small selective coefficients. Thus bristle numbers can change only very slowly by natural selection.

\section{Summary}

1. Mathematical models are described that relate the fitness of the individual to the phenotypic values of particular characteristics. In the most generally useful model, fitness is assumed to decrease as the square of the deviation of the character from an optimum value. It is shown that this model can be fitted to data of the changes of mean and variance of the character after selection.

2. If the proportions of each phenotype (or the proportions in each range of phenotypic values) are known before and after selection, the relative fitnesses can then be calculated directly. 
3. Dowdeswell's data on selection for spot number in Maniola jurtina are used to illustrate and compare the relative fitnesses calculated directly and by the mathematical model. The model shows that the intensity of selection acting on spot number is $0 \cdot 127$.

4. In experiments using large populations of Drosophila, the absolute fitness (as a probability of survival) was calculated of individuals varying in the number of their sternopleural bristles. In two of the populations the amount of food was reduced so that competition for it was intense. Only under these conditions of intense competition did fitness vary between individuals with different numbers of bristles.

5. The mathematical model was fitted to the data of one of the Drosophila populations. It was shown that a new mutation affecting bristle number could have a selective advantage only if its effect on bristle number was small.

6. Since mutations having large effects would rapidly be eliminated, mutations with small effects and low selective coefficients will make the major contribution to the variance of quantitative characters.

Acknowledgment.-The greater part of this work was done at Brown University with the financial help of a grant No. GB8677, from the National Science Foundation of America.

\section{REFERENGES}

CREED, E. R., DOWDESWELl, W. H., FoRd, E. B. and MCWHIRTER, K. G. 1959. Evolutionary studies on Maniola jurtina: the English mainland, 1956-1957. Heredity, 13, 363-391.

DOWDESWELL, w. H. 1961. Experimental studies on natural selection in the butterfly, Maniola jurtina. Heredity, 16, 39-52.

FISHER, R. A. 1930. The Genetical Theory of Natural Selection. Clarendon Press, Oxford. Fisher, R. A. 1954. Statistical Methods for Research Workers, 12th edition. Oliver and Boyd, Edinburgh.

haldane, J. B. s. 1954. Proc. Ninth Intern. Congr. Genet., 1, 480.

o'DONALD, P. 1968. Measuring the intensity of natural selection. Nature, 220, 197-198.

VAN VALEN, L. 1965. Selection in natural populations. III. Measurement and estimation. Evolution, 19, 514-558.

VAN VALEN, L. 1967. Selection in natural populations. 6. Variation genetics and more graphs for estimation. Evolution, 21, 402-405.

wooLf, B. 1955. On estimating the relation between blood group and disease. Ann. Human Genetics, 19, 251-253. 